\title{
SENTINEL-1 FDBAQ PERFORMANCE VALIDATION USING TERRASAR-X DATA
}

\author{
Elke Malz \\ Ilmenau University of Technology \\ Electronic Measurement Lab \\ 98693 Ilmenau \\ elke.malz@tu-ilmenau.de
}

\author{
Rolf Scheiber \\ Josef Mittermayer \\ German Aerospace Center DLR \\ Microwaves and Radar Institute \\ 82230 Oberpfaffenhofen \\ rolf.scheiber@dlr.de
}

\author{
Paul Snoeij \\ Evert Attema \\ European Space Agency ESA- \\ ESTEC \\ 2201 Noordwijk \\ paul.snoeij@esa.int
}

\begin{abstract}
Two Block Adaptive Quantization (BAQ) algorithms considered for implementation on-board Sentinel-1, the Entropy Constrained BAQ (ECBAQ) and the Flexible Dynamic BAQ (FDBAQ) are investigated with real data acquired by TerraSAR-X. The two algorithms are compared with respect to the resulting signal-to-quantization-noise ratio (SQNR) and the compression rate. The results confirm the improved performance of FDBAQ to be expected for Sentinel-1 compared to the more conventional ECBAQ.
\end{abstract}

Index Terms-Block adaptive quantizer, quantization, data compression, synthetic aperture radar (SAR).

\section{INTRODUCTION}

SAR satellite data provide a wide range of applications. Technically the recent SAR sensors provide large bandwidth, high sensitivity, multiple operating modes and increased operation time in orbit. Future digital beam forming promises much wider swaths leading to a further increase of the payload data rate. This forces a better on-board raw data compression since the data downlink remains often the bottleneck of a satellite SAR mission.

The conventional data compression technique applied to SAR systems is often the block adaptive quantization BAQ [1], but future SAR missions require methods with better compression performance. Beside vector quantization methods [2] and Trellis coding [3] there are also effective BAQ algorithms, like the Entropy Constrained BAQ (ECBAQ), [4,5] or the newly introduced Flexible Dynamic BAQ (FDBAQ), $[6,7,8]$ selected for implementation on-board Sentinel-1 [6].

In this paper the anticipated FDBAQ performance in terms of SQNR and compression rate based on simulation is confirmed using real satellite data and compared to ECBAQ. Essential for this investigation was the availability of high dynamic range (8bit) data from TerraSAR-X.

\section{ALGORITHM DESCRIPTION}

\subsection{Entropy Constrained Block Adaptive Quantization}

The Entropy Constrained BAQ is a widely discussed BAQ algorithm in the field of Synthetic Aperture Radar. There are a lot of variants of this algorithm, which are e.g. described in [4, 5]. Within this work we have used the algorithm as it is described in [4], which consists of a uniform BAQ followed by an Entropy coder combined with a step size control. An Entropy coder is a lossless coder; here we use a Huffman coder. The BAQ coding is performed in the time-domain. The step size control corresponds to a scaling of the input data of the individual block to a reference standard deviation $\sigma_{r e f}$.

The combination of a uniform BAQ with a Huffman coder was shown to be the optimum quantizer $[4,5]$. The approximate average distortion achievable by this combination is comparable to the Shannon optimum performance, as given by the distortion rate bound [5]. The block diagram of the ECBAQ is given in Fig. 1. Inputs are the block length BL, necessary for the extraction and recombination of the individual blocks, as well as the bitrate $\mathrm{R}_{\mathrm{BAQ}}$, which is fixed for the whole acquisition.

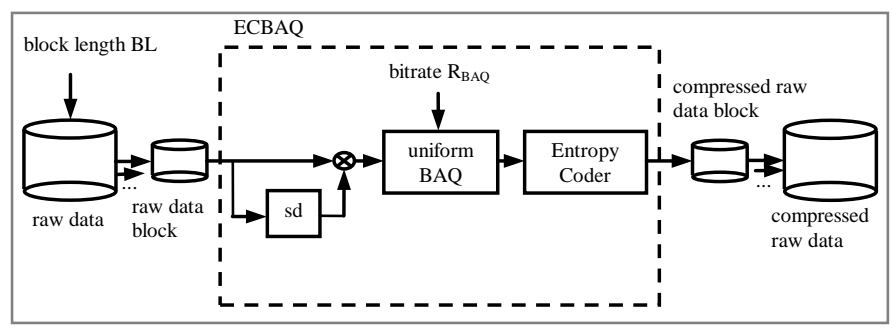

Fig. 1. Block diagram of the ECBAQ.

\subsection{Flexible Dynamic Block Adaptive Quantization}

The FDBAQ was first introduced in [7]. It is a BAQ which can have uniform or non-uniform spacing between thresholds followed by an Entropy coder, see [7, 8]. Since the described ECBAQ already defines an optimum quantizer, for the FDBAQ the same BAQ kernel as for the ECBAQ has been implemented.

In contrast to ECBAQ, the FDBAQ adapts the bitrate of every block with respect to the range location of the data block and thus with respect to the NESZ variation along range, and with respect to the mean signal backscatter, by fulfilling the socalled NESZ boundary condition. Thus, it uses a minimum number of bits and establishes a uniform NESZ along range to quantize the data. In Fig. 2, the block diagram of the FDBAQ is shown.

The range dependencies are determined by generation of the so-called range dependent scaling function $\eta(r)$. The range dependent scaling function is sensor dependent and given in Section 3 for the implementation with TerraSAR-X real test data. Given optimized quantization thresholds (defined for uniform (or non-uniform) quantization according to the maximum/minimum tolerable SQNR) and a backscatter model are used to calculate the minimum bitrate, which fulfills the NESZ boundary condition. According to the 5 discrete bitrates 


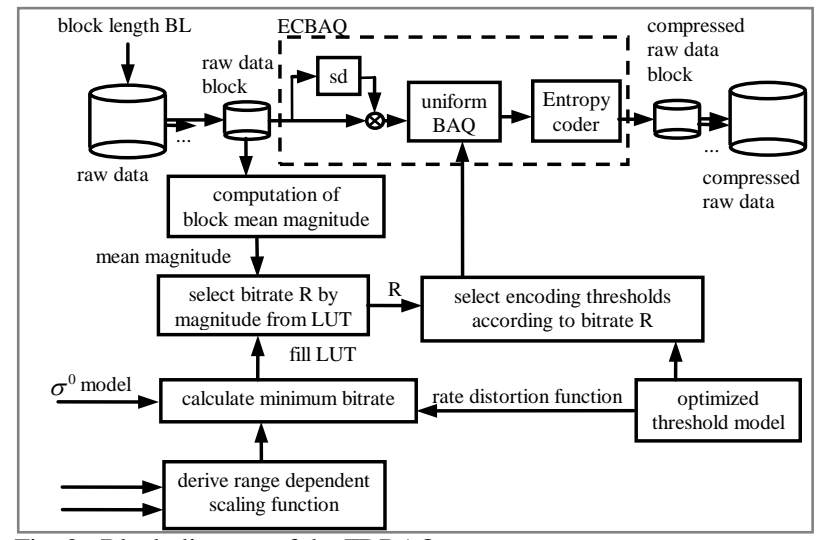

Fig. 2. Block diagram of the FDBAQ.

and the measured mean magnitude of the individual raw data block, which corresponds to the mean backscatter of the block, the bit rate is selected and the according quantization thresholds are applied. For fast on-board quantization precalculated Look-Up-Tables can be used. A BAQ that applies a constant bitrate over the whole scene independent of the input radar signal power introduces a degradation of the SNR since the SNR varies with the power of the detected radar signal [8]. Using the FDBAQ the bitrate will be controlled in a flexible way as a function of the input radar signal power. By allocating more bits to high signal levels, the SQNR can be improved resulting in an improved NESZ in the final image. This can be performed by controlling the quantization noise $N_{Q}$ in order to keep the total noise power always below a threshold, which is defined by the tolerable noise at the NESZ condition:

$\frac{N_{T}+N_{Q}}{\eta(r)}=\sigma^{0}=N E S Z \leq f$

where $N_{T}$ denotes the thermal noise, $\eta(r)$ is the range dependent scaling function, $\sigma^{0}$ is the backscatter coefficient, $f$ denotes the NESZ boundary condition and $N_{Q}$ is the maximum tolerable quantization noise power.

\section{RANGE DEPENDENT SCALING FUNCTION}

Within the range dependent scaling function (RDSF) all range variant and mission specific parameters are enclosed.

Starting from the radar equation of distributed targets [9] and using the relation $\theta_{a z} \cong \lambda / L_{a z}$ for the azimuth beam width, the RDSF results in:

$\eta(r)=\frac{P_{t} \cdot \lambda^{3} \cdot c \cdot \tau_{p} \cdot G_{2 w a y, r g}(\varphi(r)) \cdot \Delta G_{2 w a y, a z}}{2 \cdot(4 \pi \cdot r)^{3} \cdot L_{a t m} \cdot \sin \left(\eta_{\text {earth }}(\varphi(r))\right) \cdot L_{a z}}$

where $P_{t}$ is the transmit power, $C$ is the speed of light, $\tau_{p}$ is the pulse duration, $L_{a t m}$ is the loss due to atmospheric attenuation, $G_{2 \text { way,rg }}$ is the two-way gain in range, $\Delta G_{2 w a y, a z}$ denotes the degradation due to azimuth integration and azimuth steering and $L_{a z}$ is the azimuth antenna length. The parameter $\eta_{\text {earth }}$ denotes the local incidence angle on Earth and $\varphi(r)$ is the off-Nadir angle of the antenna.

\section{BAQ COMPARISON USING TERRASAR-X DATA}

In Fig. 3 the BAQ comparison environment for Sentinel-1 requirements by using TerraSAR-X test data is given.

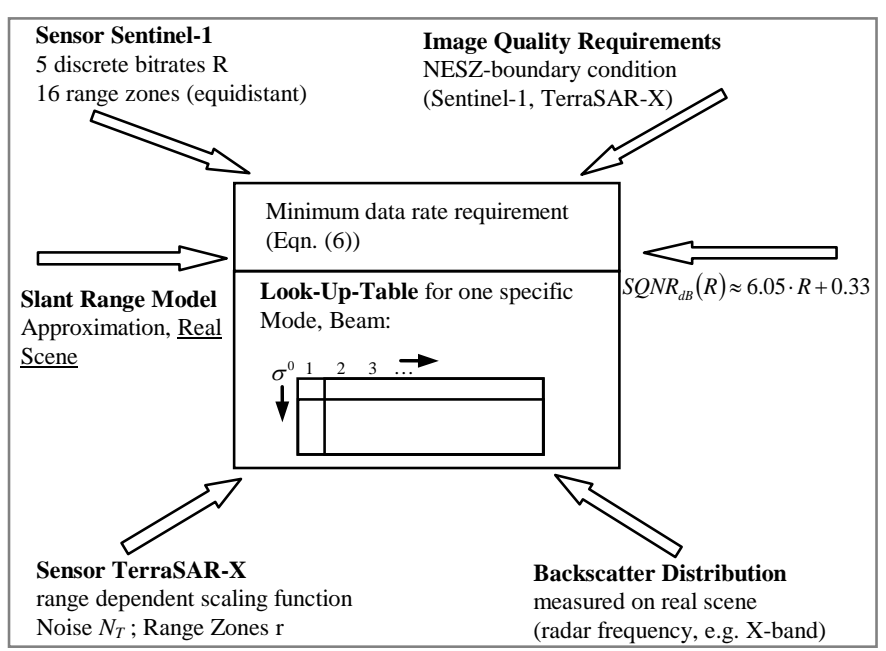

Fig. 3. FDBAQ implementation definition derived from Sentinel-1 requirements for TerraSAR-X data quantization.

There are Sentinel-1 specific sensor requirements:

- 5 discrete bitrates, due to limitations of on-board electronics of Sentinel-1

- 16 range zones, equidistantly distributed over range Furthermore we have to take into account TerraSAR-X specific sensor requirement [10]:

- the range dependency, given by the RDSF $\eta(r)$

- the thermal noise $N_{T}$

- the range zones $r$

Additionally a slant range model is required to simulate the incidence angle variation on Earth and the resulting slant range variation, which both are needed to determine the RDSF. The slant range model can be defined by approximation, or directly using the geometry of the real scene.

Further we have the Image Quality requirement, i.e. the NESZ boundary condition. The relation between bitrate and SQNR is defined by the rate distortion function, which has been generally shown to be a linear function of the bitrate $R$ after Entropy coding [5]:

$$
S Q N R_{d B}=6.05 \cdot R+0.33
$$

The coefficients of Eqn. (3) were determined by experimentally evaluating non-quantized TerraSAR-X data with five different quantizers ( $\mathrm{R}$ between 2.49 and 4.19) with given quantization threshold values, according to the selection for implementation onboard Sentinel-1. Furthermore, the mean backscatter was calculated from the measured mean magnitude of the individual raw data block. The SQNR is generally defined as:

$$
S Q N R=\frac{S+N_{T}}{N_{Q}}=\frac{\sigma_{0}+N_{T} / \eta(r)}{N_{Q} / \eta(r)}
$$

Combining Eqn. (1) and Eqn. (4) defines the boundary condition for the tolerable minimum SQNR:

$$
\operatorname{SQNR}_{d B}(R) \geq 10 \cdot\left(\log _{10}\left(\sigma_{0}+\frac{N_{T}}{\eta(r)}\right)-\log _{10}\left(f-\frac{N_{T}}{\eta(r)}\right)\right)
$$

And taking the rate distortion function (Eqn. (3)) into account, the boundary condition for the bitrate, which is the basic function for the bitrate selection in the FDBAQ algorithm, can be written as:

$$
R\left(\eta(r), \sigma_{0}\right) \geq \frac{\log _{10}\left(\sigma_{0}+\frac{N_{T}}{\eta(r)}\right)-\log _{10}\left(f-\frac{N_{T}}{\eta(r)}\right)-0.033}{0.605}
$$




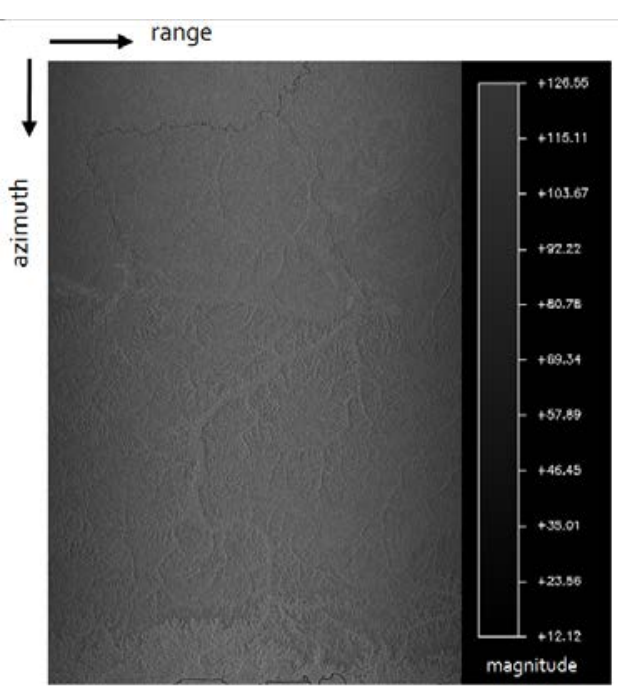

Fig. 4. TerraSAR-X test scenarios: (a) Amazonian Rainforest

Using this equation, the bitrate is calculated for different range zones, and different radar backscatter, which is related to the mean magnitude. In the Sentinel-1 mission, an optimization of the BAQ quantizers has been performed using a backscatter distribution model. Sentinel-1 uses 16 different uniformly distributed range zones and the FDBAQ is implemented using 5 predefined bitrates, which were selected based on an optimization process $[8,11]$. The corresponding thresholds for each bitrate are stored on-board in Look-Up-Tables defined by the respective range zone and the radar backscatter of the individual data block, as shown in Fig. 3. For the TerraSAR-X investigation this optimization process has not been performed, as only two data sets are investigated.

\section{TEST SCENARIOS AND TEST RESULTS}

Data for two test scenarios have been acquired by TerraSAR-X, which are in plain 8-bit format, without any BAQ compression on-board. One data take (DT) is over an Amazonian Rainforest area, Fig. 4 (a), with nearly no variations of the radar backscatter coefficient, which provides the possibility to measure pure range dependencies. A second DT with high radar backscatter variation was acquired over Tokyo. The scene was chosen to cover also an urban area and having a region with water, e.g. a lake in the middle of the scene. This DT is

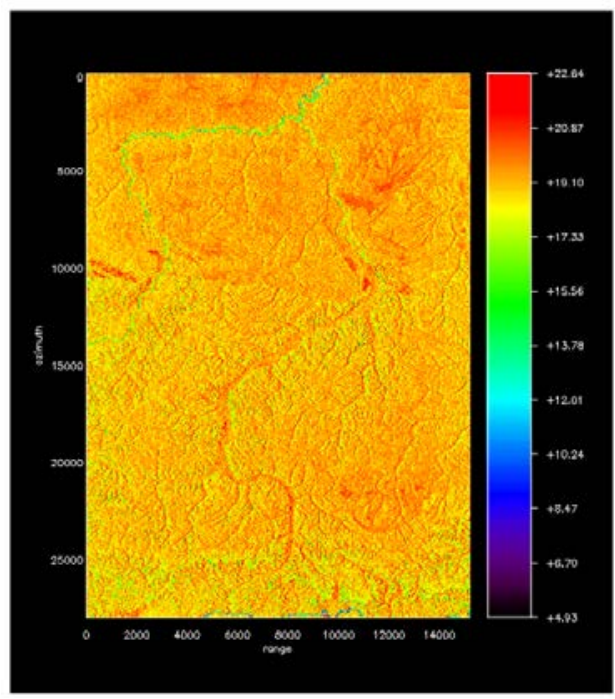

Fig. 5. SQNR map in [dB] using FDBAQ (Amazonian Rainforest test site)

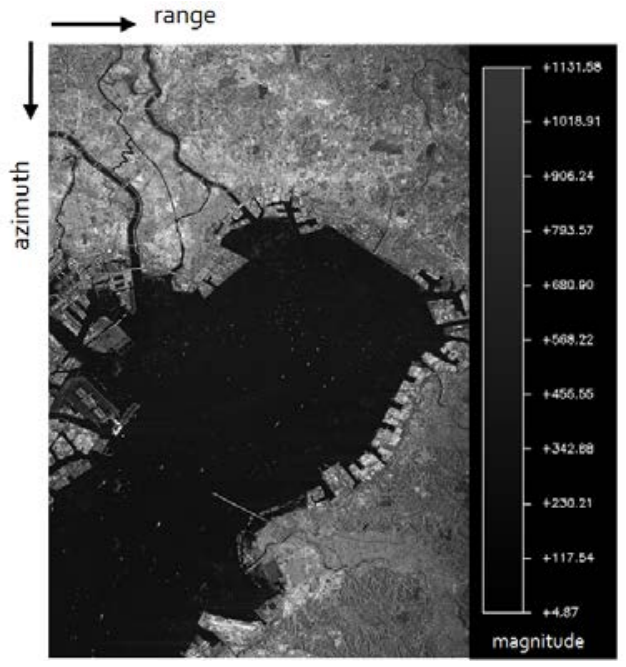

(b) Tokyo

used for the comparison of the two algorithms; see Fig. 4 (b). To visualize the regions of the image where the SQNR for both methods are different, a FDBAQ SQNR map and a difference map ECBAQ (with $\mathrm{R}_{\mathrm{BAQ}}=4$, $\mathrm{k}=8$ )-FDBAQ SQNR, was generated for the Amazonian Rainforest test site and using the ECBAQ with $\mathrm{R}_{\mathrm{BAQ}}=5, \mathrm{k}=11$ for the Tokyo test site, respectively. The SQNR maps are given in Fig. 5 and Fig. 6. Note $k$ denotes the positive number of quantization levels. The bitrate $\mathrm{R}_{\mathrm{BAQ}}$ can then be calculated by $R_{\mathrm{BAQ}}=\log _{2}(2 \mathrm{k})$. Due to the subsequent Huffman coding the effective bitrate $\mathrm{R}$ gets even smaller than $R_{B A Q}$ for the ECBAQ.

\section{COPMARISON OF ECBAQ AND FDBAQ}

The comparison is performed with respect to SQNR and compression ratio. SQNR is chosen instead of SNR to restrict the performance calculations to the contribution of the data quantization while not to emphasize the contribution of the other system parameters to the final image quality.

Table I summarizes the SQNR, the Peak SQNR as well as the mean squared error MSE for the ECBAQ and FDBAQ tests. The FDBAQ leads to a small improvement of the SQNR of about $0.1 \mathrm{~dB}$ for the Rainforest data take and of about $0.5 \mathrm{~dB}$ for the Tokyo data take. Also it can be observed that the

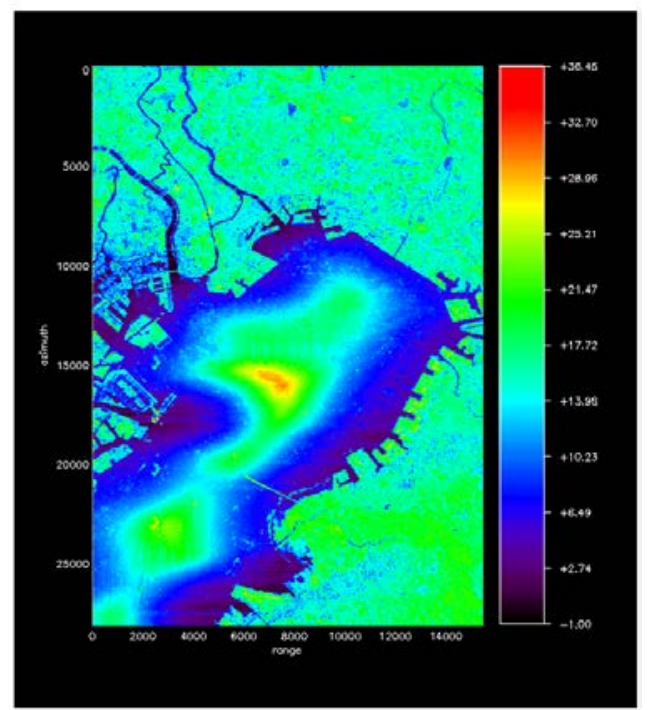

Fig. 6. SQNR map in [dB] using FDBAQ of the Tokyo test site 
TABLE I

COMPARISON OF THE SQNR OF ECBAQ AND FDBAQ

\begin{tabular}{|c|c|c|c|c|c|c|}
\hline & & $\begin{array}{l}\text { bitrate R } \\
\text { [bit/sample] }\end{array}$ & $\mathrm{k}$ & MSE & $\begin{array}{l}\text { SQNR } \\
{[\mathrm{dB}]}\end{array}$ & $\begin{array}{l}\text { PSQNR } \\
{[\mathrm{dB}]}\end{array}$ \\
\hline \multirow{10}{*}{ 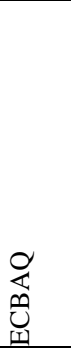 } & & 2.49 & 4 & 210.85 & 15.46 & 38.41 \\
\hline & & 2.97 & 6 & \begin{tabular}{|l|}
105.32 \\
\end{tabular} & 18.48 & 41.43 \\
\hline & 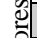 & 3.33 & 8 & 64.43 & 20.61 & 43.56 \\
\hline & 율 & 3.72 & 11 & 37.10 & 23.01 & 45.96 \\
\hline & 鳬 & 4.19 & 16 & 20.11 & 25.67 & 48.62 \\
\hline & & 2.53 & 4 & 135.94 & 15.62 & 84.22 \\
\hline & & 3.02 & 6 & 68.39 & 18.61 & 87.21 \\
\hline & & 3.36 & 8 & 41.99 & 20.72 & 89.33 \\
\hline & 간) & 3.75 & 11 & 24.32 & 23.09 & 91.70 \\
\hline & 을 & 4.24 & 16 & 13.02 & 25.81 & 94.41 \\
\hline \multirow{2}{*}{ 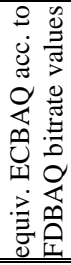 } & 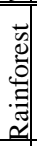 & 3.41 & 8.23 & $n A$ & 20.83 & $n A$ \\
\hline & 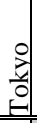 & 3.78 & 11.19 & $n A$ & 23.19 & $n A$ \\
\hline \multirow[b]{2}{*}{$\begin{array}{l}\text { 省 } \\
\text { 它 }\end{array}$} & 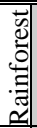 & 3.41 & 8.23 & 64.42 & 20.92 & 43.56 \\
\hline & 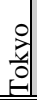 & 3.78 & 11.19 & 23.27 & 23.68 & 91.87 \\
\hline
\end{tabular}

FDBAQ allocates more bits to the Tokyo data take because of the higher backscatter, i.e. higher data rates are used. The values for the equivalent ECBAQ in terms of bitrate, listed in Table I, are determined by using the rate distortion function, Eqn. (3). The grey high lightened rows show the examples, which are discussed in Section 5.

The compression ratios obtained with ECBAQ and FDBAQ including Huffman coding are listed in Table II.

TABLE II

COMPRESSION RATIOS OF ECBAQ AND FDBAQ INCL. HUFFMAN CODER

\begin{tabular}{|c|c|c|c|c|c|}
\hline & & $\mathrm{k}$ & \begin{tabular}{|l|} 
bitrate R \\
[bit/sample]
\end{tabular} & $\begin{array}{l}\text { BAQ comp. } \\
\text { ratio }\end{array}$ & $\begin{array}{l}\text { BAQ + Huffman } \\
\text { comp. ratio }\end{array}$ \\
\hline \multirow{5}{*}{ 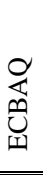 } & & 4 & 2.53 & $37.50 \%$ & $31.69 \%$ \\
\hline & & 6 & 3.02 & $44.81 \%$ & $37.74 \%$ \\
\hline & & 8 & 3.36 & $50.00 \%$ & $42.04 \%$ \\
\hline & & 11 & 3.75 & $55.74 \%$ & $46.87 \%$ \\
\hline & & 16 & 4.24 & $62.50 \%$ & $52.92 \%$ \\
\hline \multirow[b]{2}{*}{ 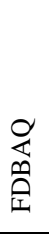 } & 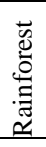 & 8.23 & 3.41 & $50.51 \%$ & $42.63 \%$ \\
\hline & 胥 & 11.19 & 3.78 & $56.05 \%$ & $47.25 \%$ \\
\hline
\end{tabular}

After compression a data volume reduction by $37 \%$ to $62 \%$ of the input data can be achieved by the uniform BAQ. The additional Huffman coding introduces an additional compression of about 15\%. Applying the FDBAQ a total compression of $42 \%$ for the Amazonian Rainforest test site and $47 \%$ for the Tokyo test site, respectively, can be observed. Note that FDBAQ allocates more bits to the urban scene to maintain the same NESZ image quality.

\section{CONCLUSION}

Two BAQ algorithms, ECBAQ and FDBAQ have been analyzed. A test approach has been implemented for TerraSAR-
$\mathrm{X}$ test data compatible with the Sentinel-1 requirements. Both BAQ algorithms have been compared regarding the SQNR performance and compression ratio. If identical equivalent compression ratios are selected for ECBAQ and FDBAQ, for the Rainforest DT an average SQNR improvement of about 0.1 $\mathrm{dB}$ was determined, which was mostly in near and far range regions. For the Tokyo DT the average SQNR improvement was about $0.5 \mathrm{~dB}$ mainly generated in the urban area and in near and far range regions. In the urban area an improvement of more than $2 \mathrm{~dB}$ was observed.

Because of its automatic adjustment of quantization parameters and therefore lowest averaged data rates for long systematic data takes, the FDBAQ has been selected for the Sentinel-1 mission [6], that has not been designed to adjust (optimize) gain for individual data takes. The investigation reported in this paper has shown for the first time by using real TerraSAR-X data that there are no hidden negative performance impacts of the FDBAQ on the SQNR caused by the flexible adjustment of the bitrate. On the contrary, for the same average data rates the FDBAQ slightly outperforms the ECBAQ because of its adjustment to the scene.

\section{ACKNOWLEDGEMENT}

This work was performed in the framework of the ESA contract: 22243/09/NL/JA. The authors would like to thank S. Wollstadt for the commanding of the plain 8-bit test sites using an experimental mode of TerraSAR-X and P. Prats for the support with the Experimental TanDEM-X Interferometric SAR Processor of DLR.

\section{REFERENCES}

[1] R. Kwok and W.T.K. Johnson,"Block adaptive quantization of Magellan SAR data", IEEE Trans. Geosci. Remote Sens., vol. 27, no. 4, pp. 375-383, Jul. 1989.

[2] J. M. Moureaux, P. Gauthier, M. Barlaud, P. Bellemain, "Raw SAR Data Compression Using Vector Quantization”, Int. Journal of Remote Sens., vol. 6, n. 16, 1995.

[3] J. W. Owens, M. W. Marcellin, B. R. Hunt, M. Kleine, "Compression of Synthetic Aperture Radar Video Phase History Data Using TrellisCoded Quantization Techniques”, IEEE Trans. Geosci. Remote Sens., vol. 37, pp. 1080- 1085, 1999.

[4] T. Algra, L. Bierens, "Frequency domain raw SAR data compression for multi-mode SAR instruments", National Aerospace Laboratory NLR, 2006.

[5] T. Algra, "Compression of Raw SAR Data using EntropyConstrained Quantization”, NLR-TP-2000-293, National Aerospace Laboratory NLR, 2000.

[6] E. Attema, C. Cafforio, M. Gottwald, P. Guccione, A. Monti Guarnieri, F. Rocca, and P. Snoeij, "Flexible Dynamic Block Adaptive Quantization for Sentinel-1 SAR Missions”, in IEEE Geoscience and Remote Sensing Letters, Vol. 7, no. 4, Oct 2010, pp. 766-770.

[7] P. Snoeij, E.Attema, A. Monti Guarnieri, and F. Rocca, "GMES Sentinel-1 FDBAQ performance analysis", in Proc. IEEE Radar Conf., Pasadena, CA, May 4-8, 2009, pp. 1-6.

[8] E. Attema, P. Snoeij, A. Monti Guarnieri, F. Rocca, P. Guccione. D. D'Aria, "Sentinel-1 Flexible Dynamic Block Adaptive Quantizer", Proceedings EUSAR, Aachen, Germany, pg. 344-349, June 2010.

[9] J.C. Curlander, and R.N. McDonough, "Synthetic Aperture Radar: Systems and Signal Processing”, John Wiley \& Sons, 1991.

[10] J. Mittermayer, M. Younis, R. Metzig, S. Wollstadt, J. Marquez, A. Meta, „TerraSAR-X System Performance Characterization and Verification“, IEEE Trans. Geoscience and Remote Sensing, vol. 48, no. 2, pp. 660-677, February 2010.

[11] P. Rizzoli, A. Monti Guarnieri, D. Giudici, D. D'Aria, "Performance Analysis of FDBAQ Quantizer for C-Band SAR Sensor”, CEOS SAR Calibration and Validation Workshop 2008, November 27-28, 2008 German Aerospace Center (DLR), Oberpfaffenhofen, Germany. 